Describing Compton scattering and two-quanta positron annihilation based on Compton profiles: two models suited for the Monte Carlo method

This article has been downloaded from IOPscience. Please scroll down to see the full text article.

2012 JINST 7 P07018

(http://iopscience.iop.org/1748-0221/7/07/P07018)

View the table of contents for this issue, or go to the journal homepage for more

Download details:

IP Address: 137.138.93.221

The article was downloaded on 23/07/2013 at 15:23

Please note that terms and conditions apply. 


\section{Describing Compton scattering and two-quanta positron annihilation based on Compton profiles: two models suited for the Monte Carlo method}

\section{T.T. Böhlen, ${ }^{a, b, 1}$ A. Ferrari, ${ }^{a}$ V. Patera ${ }^{c, d}$ and P.R. Sala ${ }^{e}$}

${ }^{a}$ European Organization for Nuclear Research CERN,

CH-1211, Geneva 23, Switzerland

${ }^{b}$ Medical Radiation Physics, Karolinska Institutet and Stockholm University,

Box 260 S-171 76 Stockholm, Sweden

${ }^{c}$ Dipartimento di Scienze di Base e Applicate per l'Ingegneria, La Sapienza Università di Roma,

Rome, Italy

${ }^{d}$ Istituto Nazionale di Fisica Nucleare - Laboratori Nazionali di Frascati,

Frascati, Italy

${ }^{e}$ Istituto Nazionale di Fisica Nucleare - Sezione di Milano,

Milano, Italy

E-mail: Till.Tobias.Boehlen@cern.ch

\footnotetext{
${ }^{1}$ Corresponding author.
} 
ABSTRACT: An accurate description of the basic physics processes of Compton scattering and positron annihilation in matter requires the consideration of atomic shell structure effects and, in specific, the momentum distributions of the atomic electrons. Two algorithms which model Compton scattering and two-quanta positron annihilation at rest accounting for shell structure effects are proposed. Two-quanta positron annihilation is a physics process which is of particular importance for applications such as positron emission tomography (PET). Both models use a detailed description of the processes which incorporate consistently Doppler broadening and binding effects. This together with the relatively low level of complexity of the models makes them particularly suited to be employed by fast sampling methods for Monte Carlo particle transport. Momentum distributions of shell electrons are obtained from parametrized one-electron Compton profiles. For conduction electrons, momentum distributions are derived in the framework of a Fermi gas. The Compton scattering model uses an approach which does not employ any free parameter. In contrast, a few semi-empirical approximations are included for the description of the complex physics of electron-positron annihilation resulting in acollinear photons. Comparisons of the Compton scattering model with simpler approaches illustrate the detailed accounting for shell structure effects. A satisfactory agreement is found for comparisons of both newly-developed models with experimental data.

KEYWORDS: Interaction of radiation with matter; Detector modelling and simulations I (interaction of radiation with matter, interaction of photons with matter, interaction of hadrons with matter, etc); Gamma camera, SPECT, PET PET/CT, coronary CT angiography (CTA) 


\section{Contents}

1 Introduction 1

2 Modelling of Compton scattering 3

2.1 Outline of the physics background 3

2.2 Compton scattering model 4

2.3 Sampling of electron momentum distributions 5

2.4 Evaluation of the Compton scattering model 8

3 Modelling of two-quanta $e^{+} e^{-}$annihilation $\quad 10$

$\begin{array}{ll}3.1 \text { Outline of the physics background } & 10\end{array}$

3.2 Acollinearity model 12

3.2.1 Empirical correction factors for selection of annihilation shell and momentum 13

3.2.2 Kinematics of two-quanta $e^{+} e^{-}$annihilation 14

$\begin{array}{lll}3.3 & \text { Evaluation of the Acollinearity model } & 14\end{array}$

4 Conclusions 17

A Lorentz and gauge transformations of polarized photons 18

\section{Introduction}

The atomic orbital structure and in specific the momentum distributions of the atomic electrons are well known to become apparent in Compton scattering [1] and positron annihilation in matter [2]. Compton scattering cross sections for a free target electron at rest are described by the Klein Nishina (KN) cross section [3]. Compared to the KN cross section, measured Compton scattering cross sections in matter exhibit prominent features which can be attributed to the shell structure: electron binding energies lead to a reaction suppression in case of low momentum transfers and the motion of the bound electrons causes a Doppler broadening of the scattered photon energy distribution. When positrons annihilate in matter with the atomic shell electrons, they decay mostly into two $511 \mathrm{eV}$ photons $\left(e^{+}+e^{-} \rightarrow 2 \gamma\right)$. Due to momentum conservation these photons have opposite directions in the centre-of-mass (cms) frame. However, in the laboratory frame the two annihilation photons are not exactly collinear and also their energies are slightly dissimilar. These slight dissimilarities are due to the momentum of the annihilating positron-electron $\left(e^{+} e^{-}\right)$system. While most of the positrons reach thermal energies before they annihilate, shell electrons may have significantly higher momentum and lead to measurable photon acollinearity and dissimilarity of photon energies. Physics models aiming at an accurate description of both positron annihilation in matter and Compton scattering require therefore a consideration of atomic shell structure effects. In case of Compton scattering, this is particularly true at low photon energies and for heavy nuclei 
(with tightly bound electrons). A detailed description of Compton scattering profits to all those applications where photon transport is important, including notably dosimetry, source shielding, photon detectors, medical linacs, positron emission tomography (PET) and dose monitoring with prompt photons [4]. To the contrary, angular correlation effects and Doppler broadening of twoquanta annihilation have a small impact on transport and energy depositions, therefore they are relevant only for applications that aim to observe the annihilation process itself. Most prominently this is the case for PET. The two-quanta positron annihilation is the underlying physical process which is made use of by PET to obtain information about the location of positron emitters. In the field of molecular imaging, PET is widely used to determine in vivo distributions of biologically active molecules, such as proteins, labelled with a positron emitter $[5,6]$. In recent years there is also an increased interest in employing the PET technique for dose monitoring during cancer treatment with hadron therapy $([7,8]$ and references therein). Positron annihilation spectroscopy is a further technique which makes use of two-quanta positron annihilation [2, 9-11].

This work proposes two models describing Compton scattering and acollinearity of two-quanta positron annihilation which account for atomic shell structure effects in media. The models are referred to in the following as Compton scattering model and Acollinearity model. Both models are based on the atomic shell binding energies and electron momentum distributions. Momentum distributions of shell electrons are obtained from parametrized quantum mechanical calculations. For conduction electrons, momentum distributions are obtained from the free electron gas model. The Compton scattering model uses an approach which does not employ any free parameter. In contrast, a few semi-empirical approximations are included for the description of the complex physics of electron-positron annihilation resulting in acollinear photons. The presented models were designed and developed for the use as native models of the Monte Carlo (MC) particle transport code FLUKA $[12,13]$. They are well suited for MC calculations, as they allow the fast sampling from the required probability density functions (PDF). FLUKA is a multi-purpose code which is capable of handling about 60 different elementary particles, including photons, leptons, hadrons and also ions, at energy ranges from a few $\mathrm{keV}$ to thousands of $\mathrm{TeV}$ in complex geometries and compound media. Neutrons are transported down to thermal energies. In specific, FLUKA simulates photons, electrons and positrons including models for all significant interaction processes at the given energy range. For photons this includes: pair production, Compton scattering, Photoelectric effect, Rayleigh scattering, photomuon and photohadron production. Photons are transported taking into account photon polarization for the Compton, the Rayleigh and the photoelectric effect. For electrons and positrons this includes: Coulomb scattering (single and condensed multiple scattering), continuous energy loss, including energy loss straggling and, above a given threshold, discrete energy losses and explicit delta-ray production via Bhabha and Møller scattering, Bremsstrahlung, positron annihilation in flight and at rest. For both stopping power and Bremsstrahlung, differences between electrons and positrons are taken into account. Atomic electron excitations or vacancies are treated by modelling de-excitation cascades leading to the subsequent emission of $\mathrm{x}$-rays, and Auger electrons. Also the production of unstable nuclei and the time evolution and tracking of radiation emitted by these residual nuclei can be performed. More details about FLUKA can be found at [14].

The article is structured as follows. Section 2 presents the Compton scattering model. Section 3 is dedicated to the description of $e^{+} e^{-}$annihilation and the Acollinearity model. Each of 
the sections contain an outline of the physics of the respective process with the focus set on the description of effects due to the binding and motion of the atomic electrons (section 2.1 and 3.1). It is followed by the model descriptions (section 2.2 and 3.2) and validations in comparison with other modelling approaches and experimental data (section 2.4 and 3.3). The procedure for obtaining the PDF of the electron momentum distributions which are required by the models is described in section 2.3 .

\section{Modelling of Compton scattering}

\subsection{Outline of the physics background}

The incoherent scattering process, also called Compton scattering, is the dominant interaction type for photons in the energy range between some $\mathrm{keV}$ up to about $5 \mathrm{MeV}$. If the momentum transfer of a photon is large compared to the electron binding energies of the target atoms, Compton scattering can be described with good accuracy in the free electron approximation. For a free electron at rest, the scattering cross section is given by the well-known $\mathrm{KN}$ formula [3, 15]

$$
\left(\frac{\mathrm{d} \sigma}{\mathrm{d} \Omega}\right)_{\mathrm{KN}}=\frac{r_{0}^{2}}{4} \frac{k^{\prime 2}}{k^{2}}\left(\frac{k}{k^{\prime}}+\frac{k^{\prime}}{k}-2+4\left(\varepsilon_{\mu}^{\prime} \varepsilon^{\mu}\right)^{2}\right)
$$

Here $k$ and $k^{\prime}$ are the initial and final photon momenta, $\Omega$ is the direction of the scattered photon relative to the initial photon, and $r_{0}=\alpha \hbar / m_{\mathrm{e}} c=2.82 \cdot 10^{-13} \mathrm{~cm}$ is the classical electron radius. For a given initial photon momentum $k$, the photon scattering angle $\theta$ and its final momentum $k^{\prime}$ are related due to energy and momentum conservation by

$$
k^{\prime}=\frac{k m_{\mathbf{e}} c}{m_{\mathbf{e}} c+k(1-\cos \theta)},
$$

the so-called Compton relation, where $m_{\mathbf{e}}$ is the electron rest mass. Again due to momentum conservation, the direction of the emitted electron is given by the direction of the momentum transfer $\mathbf{q}=\mathbf{k}-\mathbf{k}^{\prime}$. Consequently, the polar angle $\theta_{\mathbf{e}}$ is given by

$$
\cos \theta_{\mathbf{e}}=\frac{k^{\prime}+m_{\mathrm{e}} c}{k^{\prime}} \sqrt{\frac{k-k^{\prime}}{2 m_{\mathrm{e}} c+k-k^{\prime}}}
$$

and the polar angle of the electron $\phi_{\mathbf{e}}$ is opposite to the polar angle of the scattered photon $\phi$, i.e. $\phi_{\mathbf{e}}=\phi+\pi$.

$\varepsilon^{\mu}$ and $\varepsilon_{\mu}^{\prime}$ are respectively the contravariant initial and covariant final polarization four-vectors. A gauge can be chosen such that the polarization vectors have only spatial components (see appendix A) and one can obtain [15]

$$
\left(\varepsilon_{\mu}^{\prime} \varepsilon^{\mu}\right)^{2}=\left|\varepsilon^{\prime *} \cdot \varepsilon\right|^{2}=\cos ^{2} \Theta
$$

where $\Theta$ is the angle between the polarization directions of the initial photon $\varepsilon$ and the final photon $\varepsilon^{\prime}$. The scattered radiation can be conveniently expressed in terms of two linearly polarized components being parallel $(\|)$ and perpendicular $(\perp)$ to $\varepsilon[16]$. For $\perp, \cos \Theta=\varepsilon^{\prime} \cdot \varepsilon=0$. For $\|, \Theta$ can 
be expressed in terms of the scattering angle $\theta$ and the angle $\varphi$ between the scattering plane $\left(\mathbf{k}, \mathbf{k}^{\prime}\right)$ and the plane $\left(\mathbf{k}^{\prime}, \varepsilon\right)$

$$
\cos ^{2} \Theta=1-\sin ^{2} \theta \cos ^{2} \varphi .
$$

From this partition it can be seen that the $\|$-component is always more intense than the $\perp$-component.

For momentum transfers $q$ which are not large compared to the electron binding energies of the target atoms, binding energies and velocity distributions of the bound target electrons have to be taken into account for an accurate description of the Compton scattering process. The Doppler broadening, which is caused by the motion of the target electrons, leads to a broadening of the peaked energy distribution (the so-called Compton line) for a given scattering angle and the Compton cross section becomes a two-variate quantity depending on the scattering angle $\theta$ and the final photon momentum $k^{\prime}$. There are different approaches to provide a more accurate description of Compton scattering for these low momentum transfers. An approximate approach is to absorb differences with respect of the $\mathrm{KN}$ cross section in a multiplicative incoherent scattering function $S(q, Z)$, where $q$ is the scalar momentum transfer and $Z$ is the atomic number of the scatterer, so that the Compton scattering is given by

$$
\left(\frac{\mathrm{d} \sigma}{\mathrm{d} \Omega}\right)_{S(q, Z)}=S(q, Z) \cdot\left(\frac{\mathrm{d} \sigma}{\mathrm{d} \Omega}\right)_{\mathrm{KN}} .
$$

A successful historical model for light systems is the calculation of $S(q, Z)$ according to Waller and Hartree [17], and similar systematic tabulations of $S(q, Z)$, as found for instance in [18], are used nowadays by $\mathrm{MC}$ transport codes. The relativistic impulse approximation (IA), described by Ribberfors et al. [19, 20], accounts for binding effects and the target electron motion and gives an accurate description of Compton scattering also for lower photon energies (down to some tens of $\mathrm{keV})$. The IA assumes that the final electron states can be represented by plane waves. In the IA, the electron motion enters in the cross section in form of atomic shell Compton profiles (for definition see (2.9)). The adaption of the relativistic IA approach for a MC transport code is described by Brusa et al. [21].

\subsection{Compton scattering model}

A newly-developed modelling approach for Compton scattering, alternative to the ones priorly mentioned, is presented in the following. The model transforms the Compton interaction of a photon with a bound electron in motion to the simpler case of an interaction with an electron at rest. This allows to describe the scattering process itself based on the $\mathrm{KN}$ cross section (2.1). The treatment of the scattering process in the rest frame of the electron allows a significant reduction of the mathematical complexity of the problem and the related necessary computation time. Furthermore, it also allows to account for the photon polarizations accurately in a simple manner. Effects due to the motion and binding of the atomic shell electrons emerge naturally and result in scattering suppression for low momentum transfers due to energy conservation and Doppler broadening as explained in the following description of the sampling algorithm.

For a given photon in a medium with momentum four-vector $\left(k^{\mathrm{lab}}\right)^{\mu}$ and polarization $\left(\varepsilon^{\mathrm{lab}}\right)^{\mu}$, the Compton scattering interaction probability is sampled from the total $\mathrm{KN}$ cross section. The 
total Compton cross section is calculated using the electron density of the medium and assuming free electrons at rest. If a photon interacts, the target shell electron participating in the interaction is sampled from all the electrons of the medium, weighted with their relative occupation numbers ('additivity rule'). Next, the four-momentum $\left(p^{\text {lab }}\right)^{\mu}=\left(m_{\mathrm{e}} c+T_{\mathrm{e}}^{\text {lab }} / c, \mathbf{p}^{\text {lab }}\right)$ of the target electron and a corresponding kinetic energy of the target electron $T_{\mathrm{e}}^{\text {lab }}$ before the interaction is obtained. This is done by sampling the modulus of the electron momentum from the momentum distribution of the orbital (2.11), with $J$ given by (2.14), and assigning a uniformly distributed random direction. The photon four-momentum and its polarization $\left(k^{\mu}, \varepsilon^{\mu}\right)$ is then transformed by a Lorentz boost into the rest frame of the electron (for details see appendix A). In the rest frame of the electron the $\mathrm{KN}$ cross section is employed to sample the polarization $\varepsilon^{\prime \mu}$ and momentum $k^{\prime \mu}$ of the scattered photon and the related four-momentum $\left(p^{\mathrm{lab}^{\prime}}\right)^{\mu}=\left(m_{\mathrm{e}} c+T_{\mathrm{e}}^{\mathrm{lab}^{\prime}} / c, \mathbf{p}^{\mathrm{lab}}\right)^{\prime}$ of the recoiling electron. In the model the electron is considered to reside in a potential well with a depth given by

$$
V=E_{\mathrm{B}}+E_{\mathrm{H}},
$$

where $E_{\mathrm{B}}$ is the binding energy of the electron shell and $E_{\mathrm{H}}=T_{\mathrm{e}}^{\text {lab }}$ is the hole energy. FLUKA uses tabulations of atomic binding energies from [18]. In case that the kinetic energy of the recoiling electron $T_{\mathrm{e}}^{\mathrm{lab}}$ in the laboratory frame is lower than $V$, the reaction is suppressed. Instead, if $T_{\mathrm{e}}^{\mathrm{lab}^{\prime}} \geq V$ the interaction occurs and the energy $k^{\mathrm{lab}^{\prime}}$ and the direction of the scattered photon and the electron in the laboratory frame are obtained while subtracting $V$ from $T_{\mathrm{e}}^{\text {lab }}{ }^{\prime}$ for the escaping electron without modifying its direction. This is reasonable for a central potential and a good approximation if $T_{\mathrm{e}}^{\text {lab' }}$ significantly larger than $V$.

\subsection{Sampling of electron momentum distributions}

Both presented models require sampling from momentum distributions of atomic electrons. Electron momentum distributions are sampled using Compton profiles. This is done since detailed data and calculations of electronic Compton profiles are found in literature as they are an experimentally measurable quantity. The momentum density $I_{n, l}^{m}(p)$ of an atomic electron is given by [22]

$$
I_{n, l}^{m}(p)=p^{2}\left|\psi_{p}(p)\right|^{2}
$$

where $\psi_{p}(p)$ is the electron wavefunction in momentum space and $n, l, m$ are the principal, azimuthal and magnetic quantum number of the respective sub-shell. The electronic Compton profile $J_{n, l}^{m}(Q)$ is related to the momentum density $I_{n, l}^{m}(p)$ of an electron in a given atomic orbital with momentum $p$ by [22]

$$
J_{n, l}^{m}(Q)=\frac{1}{2} \int_{Q}^{\infty} \frac{I_{n, l}^{m}(p)}{p} \mathrm{~d} p,
$$

where $Q$ is given by the projection of the electron momentum $\mathbf{p}$ before collision on the negative momentum transfer $\mathbf{q}$

$$
Q=-\frac{\mathbf{p} \cdot \mathbf{q}}{q}=\frac{k k^{\prime}(1-\cos \theta)-\sqrt{m_{\mathrm{e}}^{2} c^{2}+p^{2}}\left(k-k^{\prime}\right)}{q} .
$$

Here $k$ and $k^{\prime}$ are the initial and final photon momenta. 
Hence, from (2.9) follows that the momentum density distribution can be expressed in terms of the Compton profile by

$$
I_{n, l}^{m}(p)=-2 p \frac{\mathrm{d} J_{n, l}^{m}(p)}{\mathrm{d} p} .
$$

$J_{n, l}^{m}(Q)$ is an even function and generally normalized per electron, so that

$$
\int_{0}^{\infty} 2 J_{n, l}^{m}(Q) \mathrm{d} Q=1
$$

The overall Compton profile of an atom $J^{\text {atom }}(Q)$ can be obtained by summing over all occupied shells while weighting them with the electron occupation number $N_{n, l}^{m}$ for a given sub-shell [21]

$$
J^{\text {atom }}(Q)=\sum_{n, l, m} N_{n, l}^{m} J_{n, l}^{m}(Q) .
$$

In the following, we sample the orbital electron momentum distribution from (2.11). Compton profiles of atomic electron orbitals (2.9) were parametrized from accurate quantum mechanical calculations in order to allow for fast sampling procedures. Tabulated Compton profiles of atomic orbitals obtained from Hartree-Fock wave functions by Biggs et al. [22] in the approximation of closed-shell configurations were used for the fits. The tabulations include Compton profiles of all electronic orbitals of free atoms with $1 \leq Z \leq 102$, using relativistic wave functions for elements with $Z>35$ and non-relativistic wave functions for smaller $Z$ where there is only a marginal difference between the two methods [22].

For a given sub-shell $n, l, m$, the fits to the Compton profiles $J_{n, l}^{m}(Q) \equiv J(Q)$ are obtained using a combination of five terms: a Fermi-Dirac (FD) distribution, two Gaussians (G1 and G2) and two exponentials (E1 and E2)

$$
\begin{aligned}
& J(Q)=P_{\mathrm{FD}} \cdot \frac{1}{\exp [\beta(Q-\alpha)]+1} \\
& +P_{\mathrm{G} 1} \cdot \exp \left[-\frac{1}{2}\left(\frac{Q-\mu_{1}}{\sigma_{1}}\right)^{2}\right]+P_{\mathrm{G} 2} \cdot \exp \left[-\frac{1}{2}\left(\frac{Q-\mu_{2}}{\sigma_{2}}\right)^{2}\right] \\
& +P_{\mathrm{E} 1} \cdot \exp \left[-\lambda_{1} Q\right] \quad+P_{\mathrm{E} 2} \cdot \exp \left[-\lambda_{2} Q\right],
\end{aligned}
$$

with the free parameters being $\alpha, \beta, \mu_{1}, \sigma_{1}, \mu_{2}, \sigma_{2}, \lambda_{1}, \lambda_{2}$, and the relative normalization factors $P_{\mathrm{FD}}, P_{\mathrm{G} 1}, P_{\mathrm{G} 2}, P_{\mathrm{E} 1}$ and $P_{\mathrm{E} 2}$, where due to normalization $\int_{0}^{\infty} 2|J(Q)| \mathrm{d} Q=1$ for one electron. The procedure that chooses the combination of terms for the profile fit is crucial to obtain an accurate shape for all the momentum profiles with a reduced number of parameters. For a given sub-shell, the chosen approach uses at maximum three of the five terms of (2.14) to precisely fit all profiles for all atomic numbers $Z$, with the number of knots of the profiles ranging from zero to two. The number of terms used for a given profile is driven by the shell symmetry, i.e. the number of knots of the profile distribution, while the actual choice of the analytical functions which are used in the combination (mixture of exponentials and/or Gaussians and/or Fermi-Dirac) is simply made on the basis of the best $\chi^{2}$ fit among all the different possible combinations.

Figures 1 and 2 show some examples of Compton profiles as derived from the quantum mechanical calculations by Biggs et al. [22] in comparisons with MC samples of the parametrized profiles. 


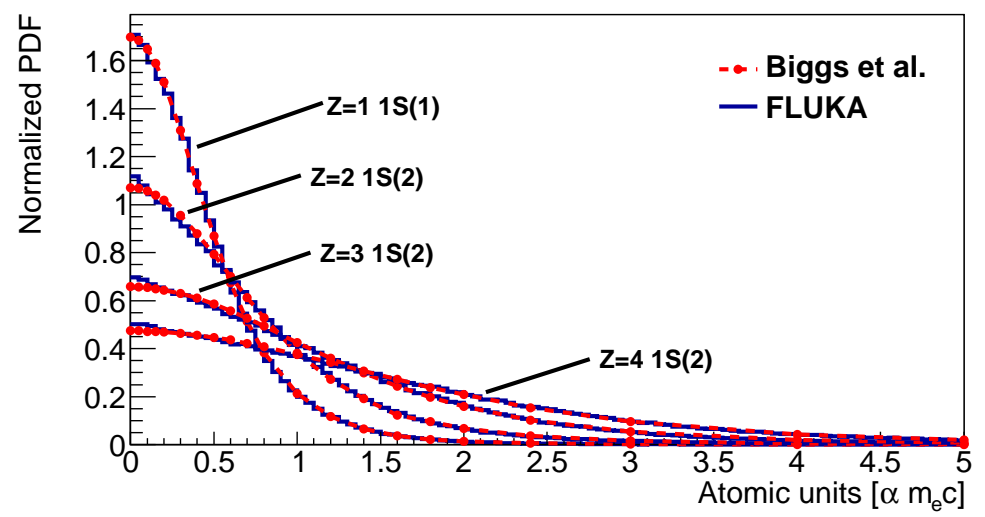

Figure 1. Examples of Compton profiles as derived from quantum mechanical calculations by Biggs et al. [22] in comparisons with parametrized MC samples. Compton profiles of the 1s orbitals of hydrogen, helium, lithium and beryllium are shown. Values in parenthesis give the shell occupation number.
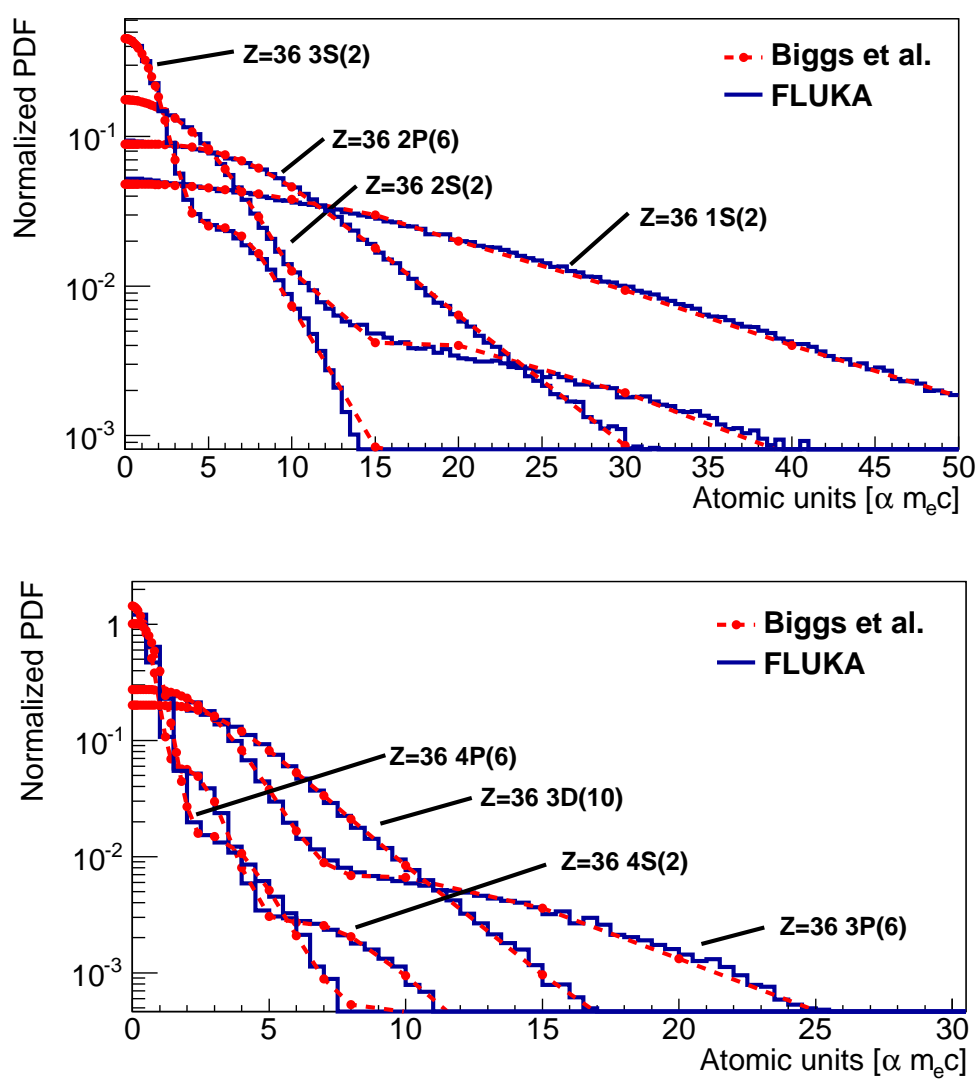

Figure 2. Examples of Compton profiles as derived from quantum mechanical calculations by Biggs et al. [22] in comparisons with parametrized MC samples. Compton profiles of the orbitals $1 \mathrm{~s}, 2 \mathrm{~s}, 2 \mathrm{p}, 3 \mathrm{~s}, 3 \mathrm{p}$, $4 \mathrm{~s}, 4 \mathrm{p}, 3 \mathrm{~d}$ of krypton are shown. Values in parenthesis give the shell occupation number. 
The momentum distribution of electrons which reside in the conduction band of a metal or semi-conductor can approximately be described in the framework of a free-electron gas with conduction band electron density $\rho_{\mathrm{e}}^{\mathrm{CB}} \cdot{ }^{1}$ The momentum distribution of a Fermi gas in zero-temperature approximation is given by:

$$
I(p)= \begin{cases}3 \frac{p^{2}}{p_{\mathrm{F}}^{3}}, & \text { if } p^{2} \leq p_{\mathrm{F}}^{2} \\ 0, & \text { if } p^{2}>p_{\mathrm{F}}^{2}\end{cases}
$$

where the Fermi momentum is given by $p_{\mathrm{F}}=h \sqrt[3]{3 \rho_{\mathrm{e}}^{\mathrm{CB}} / 8 \pi}$ with $h$ being the Planck constant. The number of conduction band electrons was chosen as found in literature.

In molecular and also in polycrystalline materials the momentum distributions of the electrons are on average isotropic. For such situations an electron momentum $\mathbf{p}=\left(p^{1}, p^{2}, p^{3}\right)$ is obtained for a given shell by sampling the modulus $p$ for the corresponding momentum distribution from (2.11) and assigning a uniformly distributed random direction.

\subsection{Evaluation of the Compton scattering model}

Figure 3 shows the total Compton scattering cross sections as a function of the initial photon energy $E$ computed with the present Compton model in comparison with the $\mathrm{KN}$ cross section and using tabulated cross sections from the Livermore Evaluated Photon Data Library 1997 (EPDL97) [18, 23]. EPDL97 cross sections are obtained by numerically integrating (2.6), that is the $\mathrm{KN}$ formula and incoherent scattering function $S(q, Z)$. For more details see also [23]. At high energies above $1 \mathrm{MeV}$, all of the approaches give almost the identical total cross section, even for heavy elements. For lower energies, differences of the $\mathrm{KN}$ cross section with respect to the other two approaches are mostly due to the cross section suppression due to shell effects compared to the free-electron picture. The EPDL97 cross sections and the present Compton scattering model account for such shell effects and their cross sections tend to zero for small photon energies $E$. In figure 4, differences between various approaches can be observed for the Compton scattering cross sections differential in energy of the scattered photon $E^{\prime}$ at selected energies $E=20,100$, $1000 \mathrm{keV}$ for carbon and lead. The shown cross sections were computed with the present Compton model, with a fit to EPDL97 tabulations, and using the KN cross section. For high energies of the scattered photon, with $E^{\prime} / E$ close to one, the approach using $S(q, Z)$ provides a smooth approximation to the shell effects, which tends to zero for $E^{\prime} / E \rightarrow 1$ where the $\mathrm{KN}$ cross section is finite. Both the $\mathrm{KN}$ cross section and the approach using $S(q, Z)$ do not account for effects due to Doppler broadening and predict a sharp fall off at $E /\left(1+2\left(E / m_{\mathrm{e}} c^{2}\right)\right)$. The Doppler broadening causes a blurring of this fall off, most notable for photon energies in the order of the kinetic energies of the shell electrons. The present Compton scattering model predicts the fine structure of differential Compton cross sections in form of discontinuities which are caused by surpassing the individual binding energies of shell electrons. It also accounts for Doppler broadening due to the motion of the target electrons. Figure 5 shows the Doppler broadening of the Compton line from measurements [24] and as predicted by the Compton scattering model at an angle of $167^{\circ}$ for $412 \mathrm{keV}$ photons incident on aluminium and germanium. There is a good agreement between simulations and measurements for both media.

\footnotetext{
${ }^{1}$ If not changed by the user, solid and liquid compounds are considered by default to be a metal in FLUKA if they consist to more than $50 \%$ (atoms) of metallic elements.
} 

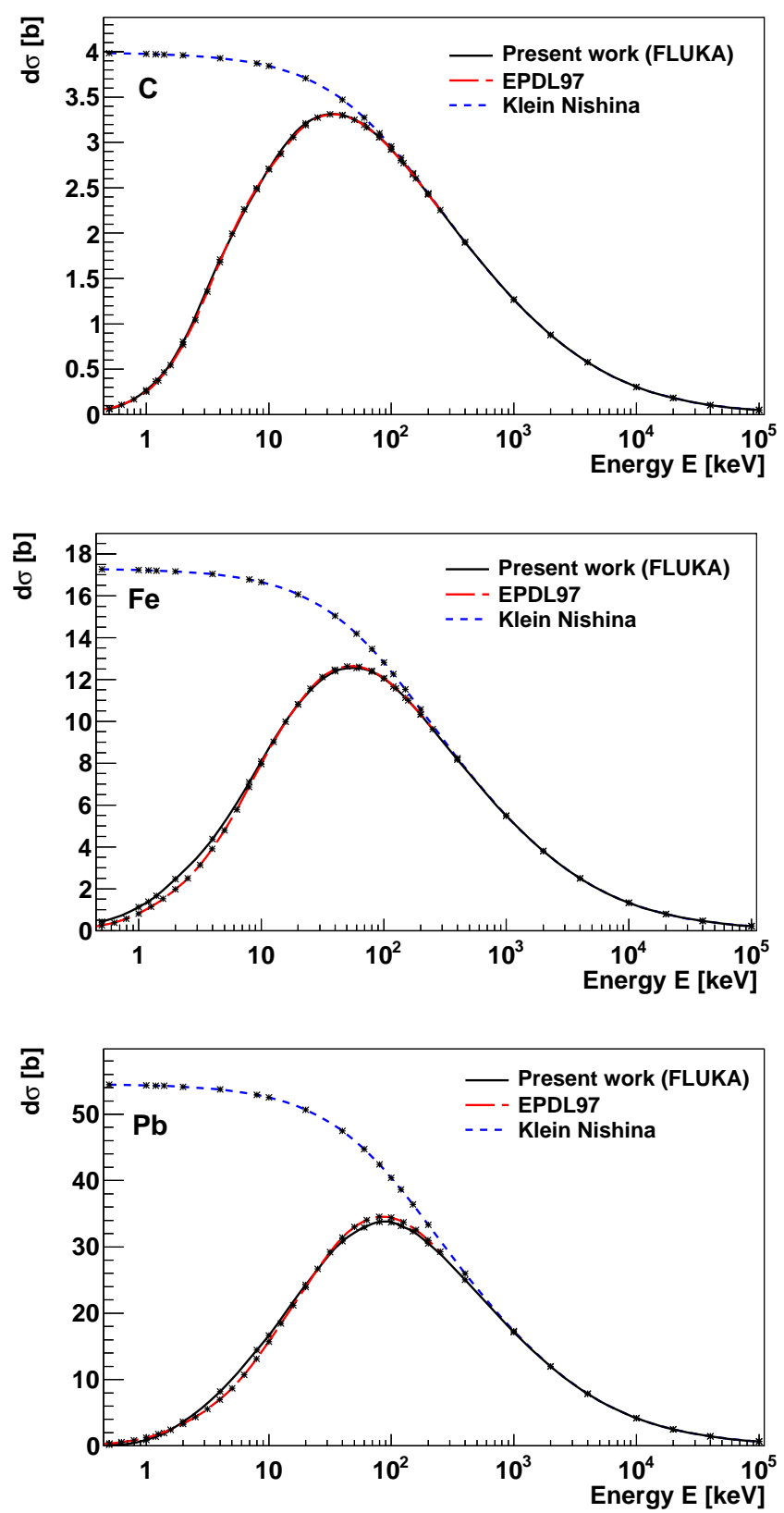

Figure 3. Total Compton scattering cross sections as a function of the initial photon energy $E$ computed with the present Compton scattering model, using cross section tabulations from EPDL97 [18], and using the KN cross section. Cross section for carbon, iron and lead are shown.

The present model describes binding effects and electron motion in the frame of closed shell atoms for orbital electrons and in the picture of a free electron gas for conduction band electrons. Chemical bonds lead to modifications of the binding energies and momentum distributions of the valence orbitals with respect to an independent atom model. This can lead to inaccuracies of the model for photon energies close to the binding energies of the outer shell electrons. 

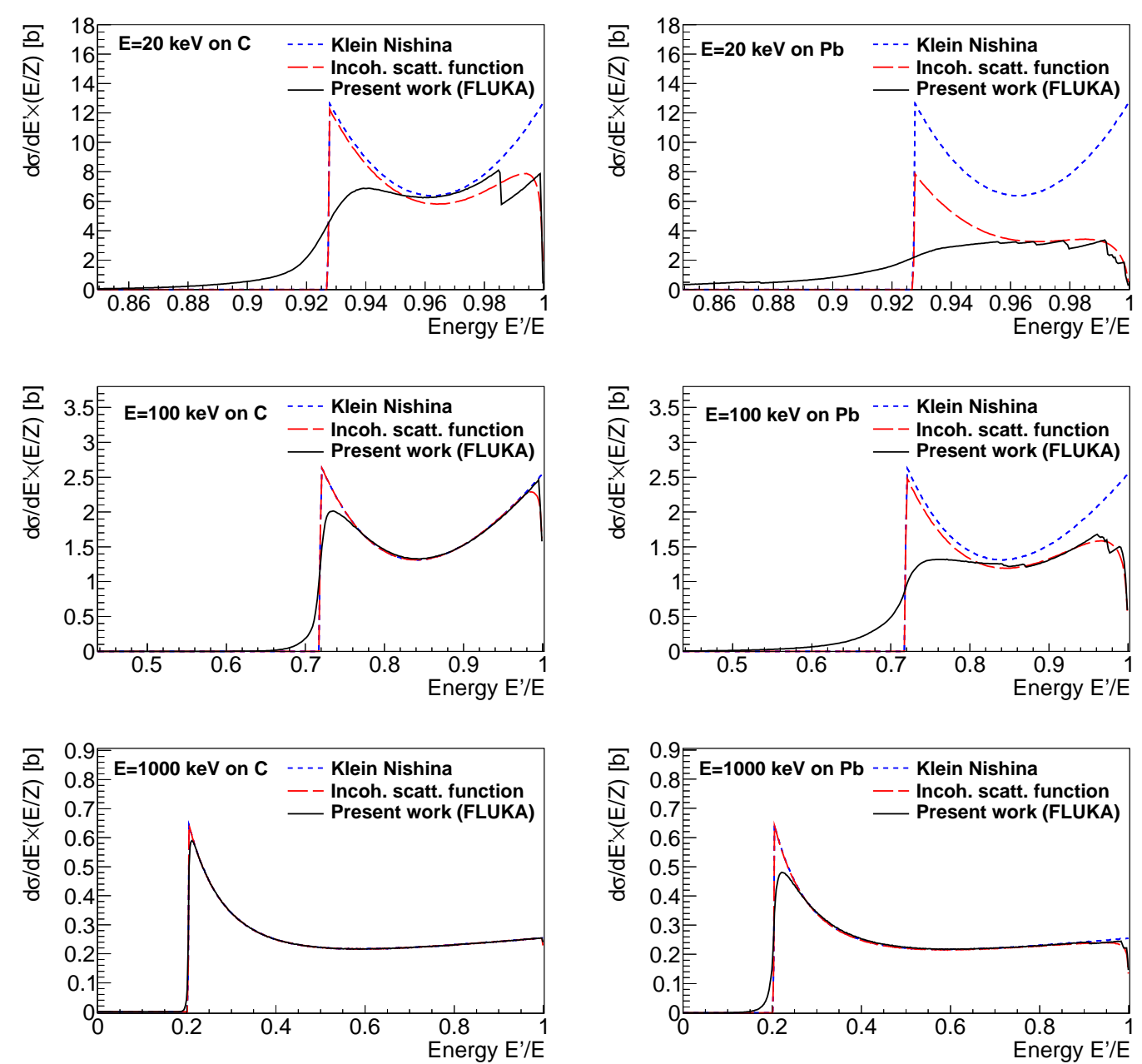

Figure 4. Compton scattering cross sections differential in the energy of the scattered photon $E^{\prime}$ at selected initial photon energies $(E=20,100,1000 \mathrm{keV})$ for carbon and lead. The cross sections are computed with the present Compton scattering model, using a fit to tabulations of the incoherent scattering function $S(q, Z)$ from EPDL97 [18], and using the KN cross section.

\section{Modelling of two-quanta $e^{+} e^{-}$annihilation}

\subsection{Outline of the physics background}

Energetic positrons in media, such as originating from positron emitters $(\sim 1 \mathrm{MeV})$, generally slow down to thermal energies (characteristic energy: $(3 / 2) \cdot k T=0.04 \mathrm{eV}$ ) due to Coulomb interaction before they annihilate. This is reflected in energy loss cross sections which are significantly larger than annihilation cross sections at higher (non-thermal) energies. For the small fraction of annihilations in flight, the momentum of the electron can be usually neglected compared to the generally much larger positron momentum. The integral and differential cross sections for two-quanta annihilation in flight of a free $e^{+} e^{-}$pair with the electron at rest can be found for instance in [16]. Annihilation in flight is readily considered by many MC codes [25], including FLUKA. Other types of annihilation, such as one-quantum, no-quantum and three-quanta annihilation, do occur. How- 

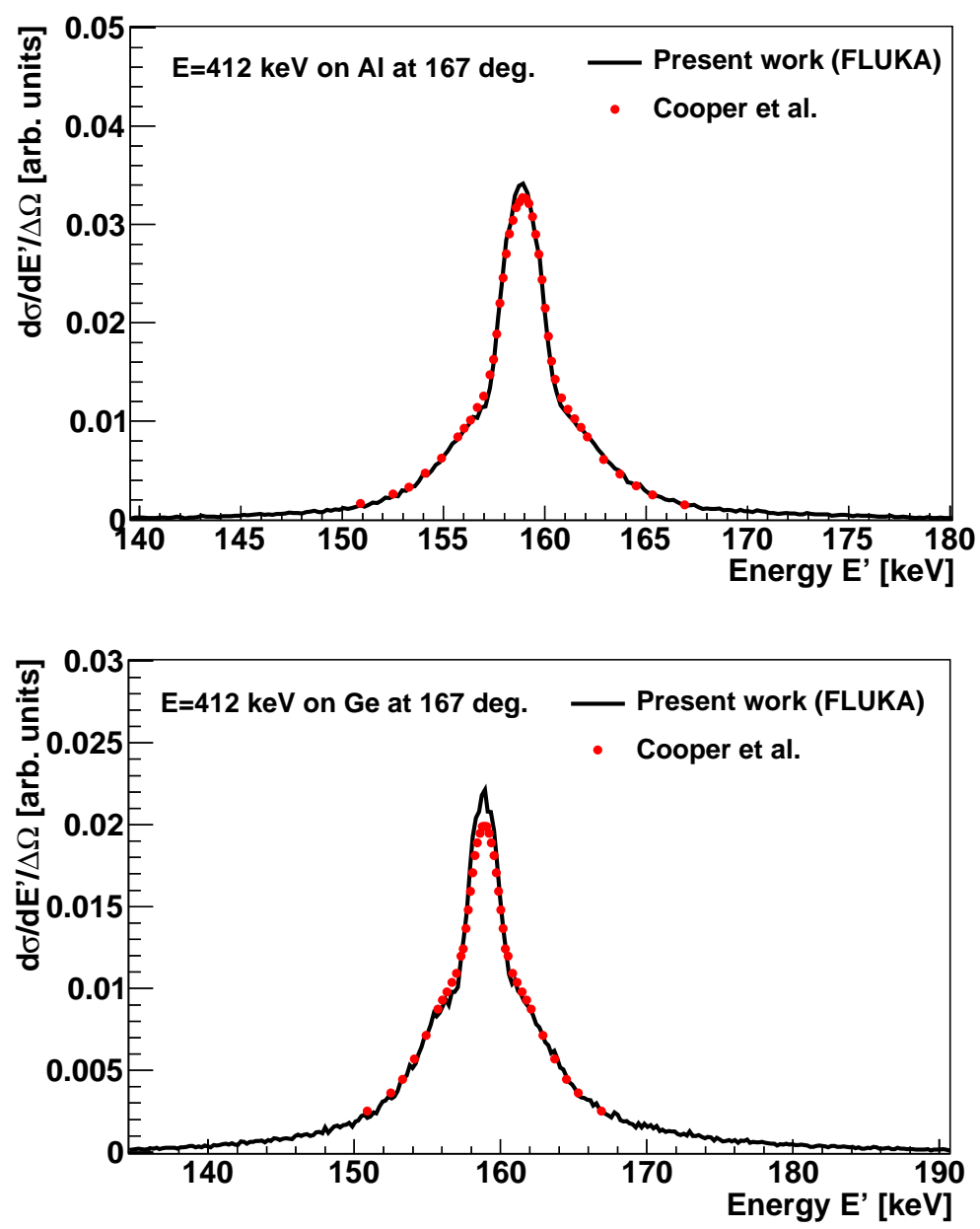

Figure 5. Compton scattering cross sections differential in the energy of the scattered photon $E^{\prime}$ at an angle of $167^{\circ}$ for $412 \mathrm{keV}$ photons incident on aluminium and germanium. Experimental data from Cooper et al. [24] are presented together with predictions of the Compton scattering model which were convolved with a Gaussian with a full-width at half-maximum (FWHM) of $0.565 \mathrm{keV}$ to imitate the detector resolution of the data [24].

ever, they are far less likely. An analytical estimation of the most probable of these processes, the one-quantum annihilation, is given by Jung [26].

The two-quanta annihilation process at rest is of particular interest for applications, such as PET, where other annihilation processes contribute merely in form of background to the measurement. For $e^{+} e^{-}$annihilations at rest, i.e. with thermalized positrons, the momentum of the annihilation $e^{+} e^{-}$pair is generally determined by the motion of the bound electron. Interactions of slow positrons with atoms, molecules, and ionic lattices are of complicated nature. The time scale of a thermal positron approaching an atom or molecule is much larger than the electron cloud relaxation time. Long-range interactions can be approximatively described by the polarization of the atom or molecule and dipole-charge interactions [27]. For short-range interactions (at distances $\sim$ Bohr radius) to the contrast, no simple multipole expansion is possible any longer, and the potential of the atomic nuclei, positronium atom formation [28, 29], and the annihilation reaction have to be 
taken into account. A detailed consideration of these processes involves the quantum mechanical calculations of many-body interactions of the positron with the atomic or molecular electrons in order to describe $e^{+} e^{-}$correlation effects $[30,31]$ and phenomena, such as positron trapping effects in lattices, which alter positron lifetimes and eventually the probability of annihilation in different sites of the atom or molecule [2,9].

The angular correlation of the annihilation photons and their emission energies (Doppler broadening) are sensitive to these underlying physics processes as they can modify notably the momentum distribution of the annihilating $e^{+} e^{-}$pairs. In fact, angular correlation and energy spectra can be used for the identification of different materials (positron annihilation spectroscopy) [10] and even for monitoring material parameters such as lattice defects, evoked for instance by compression of the material $[2,32]$. The sensitivity to modifications of atomic structure is explained by the fact that positrons annihilate predominantly with outer shell electrons [33], that are valence or conduction band electrons, which are themselves only slightly bound and involved in chemical bonds. Correlation effects between electrons and positrons generally result in increased orbital densities of electrons around the positron. In descriptions based on independent particle models, correlation effects can be accounted for by means of enhancement factors for individual annihilation sites [34]. Large relevance of correlation effects are observed especially for outer shell electrons [35].

\subsection{Acollinearity model}

In spite of the complex underlying physics processes involved in positron annihilation in media, it is experimentally found that the bulk of energy and angular distributions is readily described by the sum of two Gaussians with sigmas and weights dependent on the medium for atoms and molecules of both simple and complex structures [36]. Modifications for different media of the Gaussian-alike central part of the angular and energy spectra are mostly only moderate, whereas the high-momentum tails of the distributions (appreciable in logarithmic scale) exhibit a much stronger variation. Highly precise experiments are designed to measure tails of large angular and momenta distributions over five orders of magnitude [35]. However, these tails contribute with a negligible amount to the total annihilation reaction rate and are consequently not relevant for applications such as PET imaging techniques. These observations support the intent to describe the bulk of the angular and energy spectra of two-quanta $e^{+} e^{-}$annihilation at rest approximately by a simplified semi-empirical approach.

In the FLUKA code, fast positrons are transported while considering energy losses and in flight annihilation cross sections, as mentioned earlier, according to well-known formulas, see ([16], chapter 4 , section 21). Below $1 \mathrm{keV}$, positrons are no longer transported and their remaining kinetic energy is deposited on the spot. Then annihilation at rest occurs. For modelling of the angular distribution and the correlated energy distribution of two-quanta annihilation reactions at rest, the same electron momentum distributions as for Compton scattering are used (see section 2.3). The momentum of thermal positrons is generally small compared to the momentum of the shell electrons and is set to zero (i.e. is neglected). It is important to stress here that the chosen description for the atomic electron momentum distributions neglects any modifications of electronic orbitals due to chemical bonds and the presence of the positron. Instead, $e^{+} e^{-}$correlations are introduced by the means of empirical correction factors in the spirit of the approaches discussed by Gupta and Siegel [34] and Alatalo et al. [30]. The parameters for the empirical correction factors are obtained 
by matching energy and angular correlation spectra of annihilation photons predicted by the model to experimental data.

\subsubsection{Empirical correction factors for selection of annihilation shell and momentum}

A positron at rest will annihilate inevitably with one of the atomic or conduction band electrons of the target medium. In the model, once an electron is selected for annihilation (explained later) the momentum of the electron is sampled from the corresponding momentum distribution of the sub-shell or the free electron gas momentum distribution, in case of a conduction band electron, see (2.11), (2.14) and (2.15). To allow for a suppression of annihilations with high-momentum shell electrons, a momentum-dependent correction factor $R_{n, l}^{m}(p)$ is used. Hence, the PDF of the annihilation momentum of the $e^{+} e^{-}$pair for an electron shell $n, l, m$ is given in the model by

$$
A_{n, l}^{m}(p)=R_{n, l}^{m}(p) I_{n, l}^{m}(p) .
$$

We chose to introduce an empirical factor which matches the data of angular correlation and Doppler broadening measurements of the hydrogen electron (see figures 7 and 9). The factor, rescaled by the corresponding binding energy $E_{n, l}$, is then applied to all sub-shells of all elements. It is parametrized in the form of a double-Gaussian PDF

$$
R_{n, l}^{m}(p)=N_{n, l} \cdot\left(0.9 \cdot \exp \left[-\frac{1}{2}\left(\frac{p}{0.7 \cdot F_{n, l}}\right)^{2}\right]+0.1 \cdot \exp \left[-\frac{1}{2}\left(\frac{p}{1.5 \cdot F_{n, l}}\right)^{2}\right]\right),
$$

where $p$ is the electron momentum,

$$
F_{n, l}=\sqrt{2 m_{\mathbf{e}} E_{n, l}}
$$

and $N_{n, l}$ being the normalization factor. No momentum correction factor is used for the conduction band electrons and their momentum is directly sampled from the free electron momentum distribution (2.15).

The unnormalized relative probability $P_{n, l}^{\mathrm{e}^{-}}$for a given positron to annihilate with a certain subshell electron $n, l$ or conduction band electron is assumed to depend only on the binding energy $E_{n, l}$ of the electron and is parametrized by a Gaussian of the form

$$
P_{n, l}^{\mathrm{e}^{-}}=\exp \left[-\frac{1}{2}\left(\frac{E_{n, l}}{\sigma_{\mathrm{e}^{-}}}\right)^{2}\right]
$$

where $\sigma_{\mathrm{e}^{-}}$is a free parameter. $\sigma_{\mathrm{e}^{-}}$was determined by matching data of angular correlation measurements for nitrogen (see figure 7) and set to $23 \mathrm{eV}$. To compute $P_{n, l}^{\mathrm{e}^{-}}$for the case of annihilation with conduction electrons, conduction electrons are assumed to reside in a potential well made up of a binding energy assumed to be $2 \mathrm{eV}$ and the average kinetic energy of an electron in a free electron gas $E_{\mathrm{avg}}=3 / 5 E_{\mathrm{F}}$. The total binding energy $E_{n, l}$ of conduction band electrons used for sampling is thus given by

$$
E_{\mathrm{CB}}=2 \mathrm{eV}+2 / 5 \cdot E_{\mathrm{F}},
$$

with the Fermi energy $E_{\mathrm{F}}=p_{\mathrm{F}}^{2} /\left(2 m_{\mathrm{e}}\right)$. The normalized probability for the annihilation with a certain target electron $n, l, m$ for a given medium is

$$
\hat{P}_{n, l, m}^{\mathrm{e}^{-}}=\frac{w_{n, l, m} \cdot P_{n, l}^{\mathrm{e}^{-}}}{\sum_{n^{\prime}, l^{\prime}, m^{\prime}} w_{n^{\prime}, l^{\prime}, m^{\prime}} \cdot P_{n^{\prime}, l^{\prime}}^{\mathrm{e}^{-}}}
$$


where $w_{n, l, m}$ is the fractional frequency of the element with electron $n, l, m$ in a compound and $n, l, m$ runs over all electrons in the medium.

\subsubsection{Kinematics of two-quanta $e^{+} e^{-}$annihilation}

In the following all momenta and energies without superscript are given in the laboratory frame while momenta and energies in the cms frame of the annihilating $e^{+} e^{-}$pair are labelled with 'cms'. An $e^{+} e^{-}$pair annihilates by emitting two quanta at an angle of $180^{\circ}$ in the cms frame which are mutually polarized. The momentum of the $e^{+} e^{-}$pair is transferred to the photons leading to a deviation from collinearity in the laboratory frame. In the laboratory frame the total initially available energy is given by

$$
E_{\mathrm{ep}}=\sum_{i=\mathrm{e}, \mathrm{p}} E_{i}-E_{\mathrm{B}}-E_{\mathrm{H}}
$$

Here $E_{i}=\sqrt{\left(m_{\mathrm{e}} c^{2}\right)^{2}+\left(\mathbf{p}_{i} c\right)^{2}}$ is the initial energy of the annihilating electron or positron, with $p_{i}^{\mu}$ being the respective momentum four-vector. $E_{\mathrm{B}}$ is the binding energy of the electron and $E_{\mathrm{H}}=E_{\mathrm{e}}^{\text {kin }}$ is the kinetic energy of the bound electron (i.e., the hole state energy). $E_{\mathrm{B}}$ and $E_{\mathrm{H}}$ form the potential well in which the electron resides. The positron is assumed to be at rest with $\mathbf{p}_{\mathrm{p}}=0$ and residing in a zero potential well. The available energy is assumed to be shared equally between the two annihilation photons in the cms frame

$$
E_{\gamma, 1}^{\mathrm{cms}}=E_{\gamma, 2}^{\mathrm{cms}}=\frac{1}{2} \sqrt{s}=\frac{1}{2} \sqrt{\left(p_{\mathrm{ep}}\right)_{\mu} p_{\mathrm{ep}}^{\mu}}
$$

where $p_{\mathrm{ep}}^{\mu}=\left(E_{\mathrm{ep}} / c, \mathbf{p}_{\mathrm{ep}}\right)$, with $\mathbf{p}_{\mathrm{ep}}=\mathbf{p}_{\mathrm{e}}$. The energy and momentum of the annihilation photons in the laboratory frame is then obtained by a Lorentz transformation given by the boost four-vector $p_{\mathrm{ep}}^{\mu}$. Binding and hole state energies are generally small compared to the energy of the annihilation photons. Hence, the approximation of subtracting $E_{\mathrm{B}}$ and $E_{\mathrm{H}}$ in the cms frame should have little effect on the angular distributions.

\subsection{Evaluation of the Acollinearity model}

Contrary to the Compton scattering model, where the selection of the participating target electron is independent of its shell and binding energy, positron annihilations occur predominantly with the less tightly bound outer-shell electrons. Positron annihilation is therefore more sensitive to modifications of these orbitals with respect to the closed-shell atom picture. We found that a simple scaling factor of 1.4 between the annihilation momentum density and the unperturbed atomic electron momentum density describes very roughly experimental Doppler broadening data from Iwata [36]. The same scaling factor was also obtained recently by Green et al. [37].

Figure 6 shows angular correlation spectra of noble gases as predicted by the present model (section 3.2) and from measurements. Noble gases consist of single elements with closed shells in which the orbitals are not modified by molecular bonds. Consequently, assuming accurate measurements, the differences between measurements and predictions of the Acollinearity model can be attributed to correlations not accounted for by the empirical correction factors, to insufficiencies in their parametrization or to the limited accuracy of the fits of the Compton profiles.

For molecular compounds, the valence shell orbitals may be modified by chemical bonds. These alterations are not considered by Compton profiles which are computed for single atoms. 

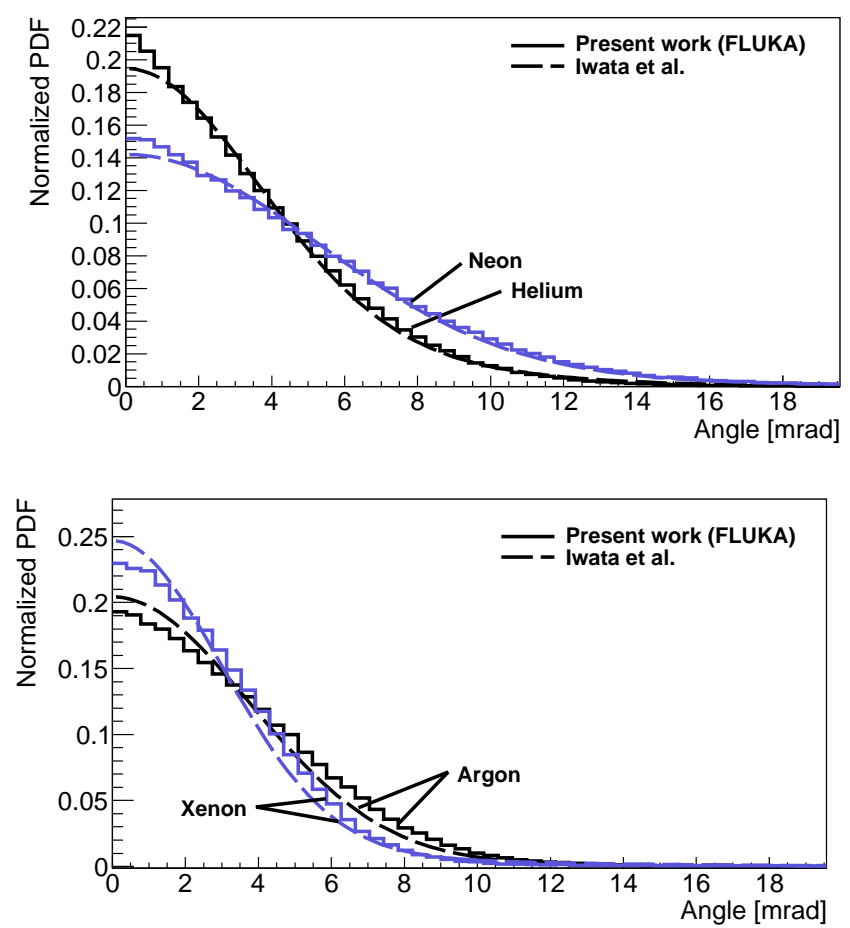

Figure 6. Simulated and measured [36] normalized angular correlation spectra for selected noble gases. Spectra show the projected (one-dimensional) angle.

Nevertheless, the agreement of the model with experimental data from simple and organic compounds is satisfactory, as shown for example in figure 7.

For metals, the central part of the angular correlation spectrum is determined by positron annihilations with conduction band electrons. In contrast, the tails of the distributions are generally dominated by annihilations with core electrons. Figure 8 shows some examples of comparisons of simulated and measured angular correlation spectra for metals. Both magnesium and calcium exhibit a clear separation between a 'central bulk'-part of the spectra produced by annihilations with conduction band electrons and a 'tail'-part produced by annihilations with the core electrons. For gold, annihilations are predicted by the model to occur only with a probability of about $10 \%$ with conduction electrons. Consequently, a strong division between 'central bulk' and 'tail'-part can not be observed. While the shape of the 'central bulk' (by conduction band electrons) and also the 'tail'-part (by core electrons) are generally well reproduced for all tested metals, the relative fraction of annihilations on each of the parts is not always in agreement with the experimental data (see for instance calcium in figure 8). The relative fraction of annihilations on the conduction band and core electrons is determined by relation (3.4) and (3.5).

In addition to the angular correlation spectrum of hydrogen, shown in figure 7, figure 9 presents the Doppler broadening spectra for hydrogen of measurements and model predictions.

The model was primarily designed to reproduce the 'bulk'-part of the angular and energy spectra. But as seen in figure 10, which shows a comparison of Doppler broadening spectra from model predictions and experimental data [38] with a logarithmic ordinate extending to low probabilities, 

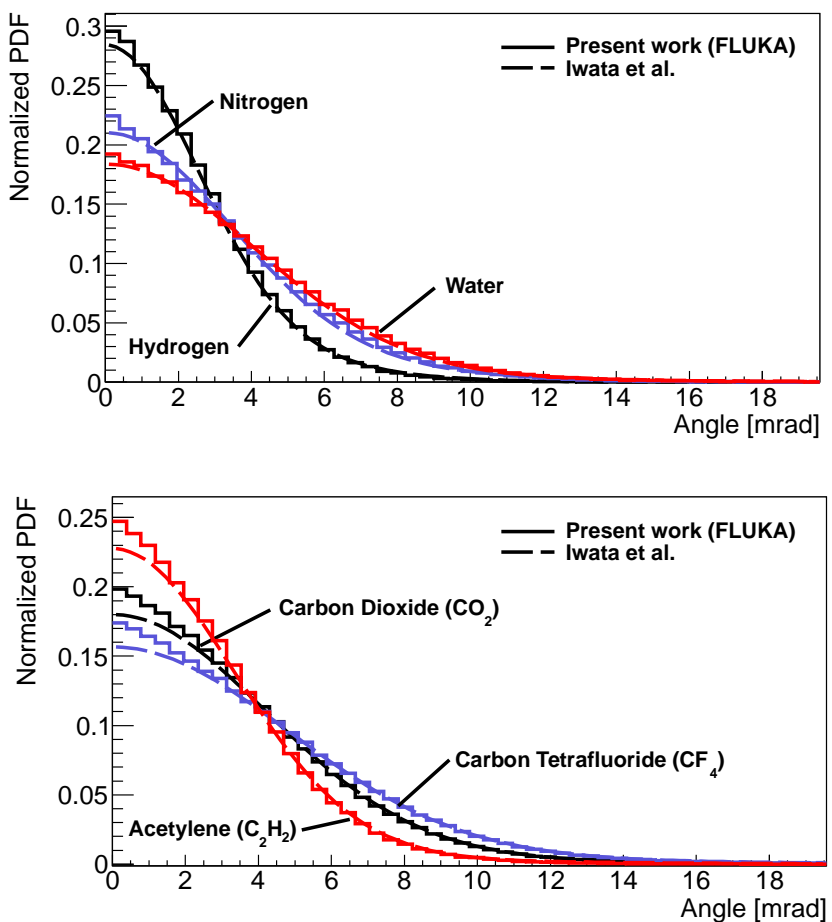

Figure 7. Simulated and measured [36] normalized angular correlation spectra for selected molecules. Spectra show the projected (one-dimensional) angle.

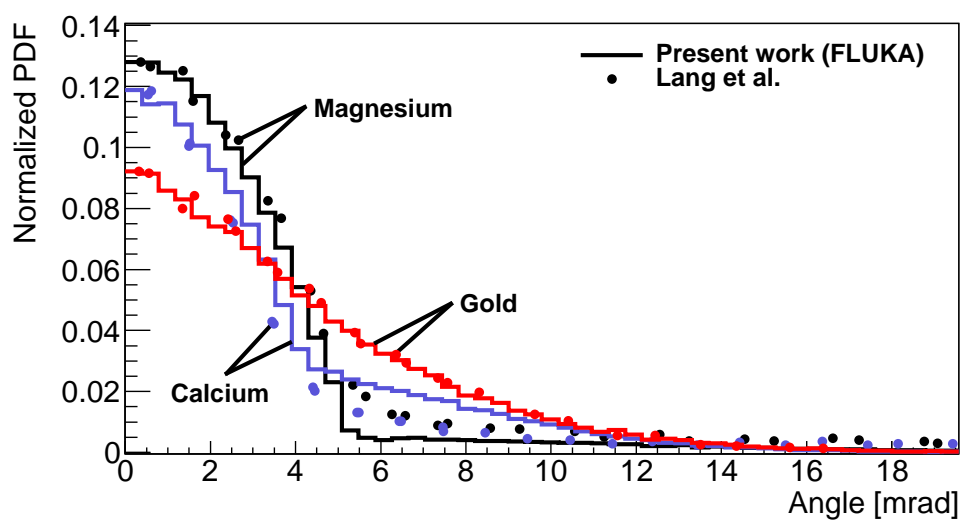

Figure 8. Simulated and measured [36] normalized angular correlation spectra for selected metals. Spectra show the projected (one-dimensional) angle.

the model does not behave badly for such low probabilities. The comparison supports the presumption that the model scheme could be used in principle as well to predict contributions to the tails of the spectra from the high-momentum core electrons down over some orders of magnitude, as used for instance by positron annihilation spectroscopy. 


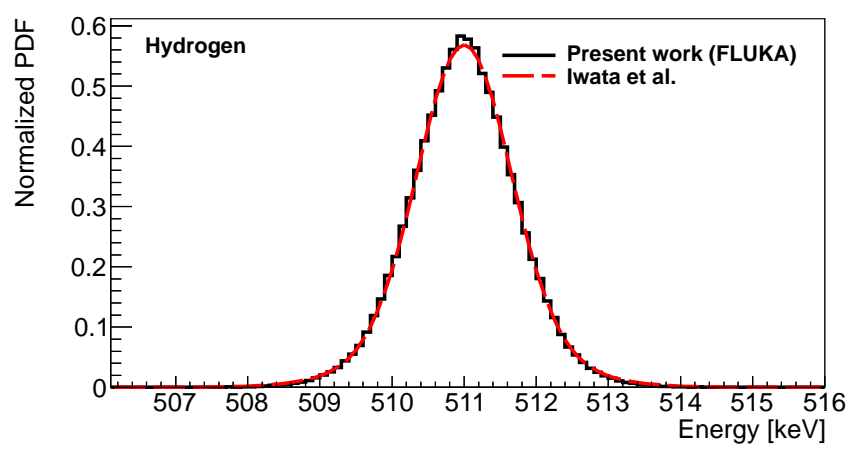

Figure 9. Simulated and measured [36] normalized energy Doppler broadening spectra for hydrogen.

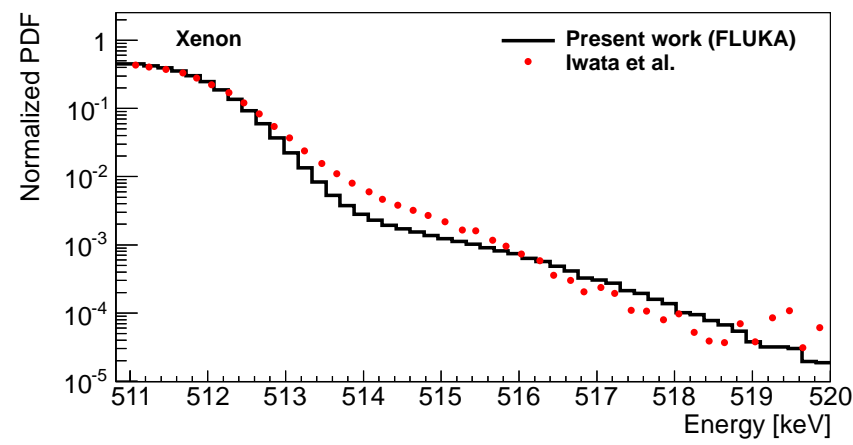

Figure 10. Simulated and measured [38] normalized energy Doppler broadening spectrum with a logarithmic ordinate.

\section{Conclusions}

Two models describing Compton scattering and acollinearity of two-quanta $e^{+} e^{-}$annihilations in matter based on electron momentum distributions were developed and integrated in the MC code FLUKA. The validation of both models shows a satisfactory agreement with experimental data. The Compton scattering model provides a detailed and parameter-free description of shell structure effects in a simple formalism while accounting for changes of photon polarization in a natural way. The Acollinearity model uses electron momentum distributions together with a semi-empirical description of $e^{+} e^{-}$correlation effects for determining the annihilation shell and momentum. Both models allow for fast MC sampling. ${ }^{2}$ For normal FLUKA simulations, where electrons, positrons and photons are transported in media, the usage of these models contributes with a negligible increase to the total simulation time. The Compton scattering model is activated by default if accurate simulations are requested in the current FLUKA version. The Acollinearity model will be active by default starting from the next FLUKA version. Activation of both models can be controlled by the user via the FLUKA input card 'EMFRAY' [14].

\footnotetext{
${ }^{2}$ The average sampling time for Compton scattering of a $511 \mathrm{keV}$ photon in lead is about $2 \cdot 10^{-6} \mathrm{~s} / \mathrm{event}$. The average sampling time for the annihilation of a positron in water is about $7 \cdot 10^{-6} \mathrm{~s} /$ event. Evaluation of the required CPU times needed for sampling are given for a processor of type: Intel(R) Xeon(R) CPU X5677@3.47GHz.
} 


\section{Acknowledgments}

The authors would like to thank Alberto Fassò for attentive proofreading of the manuscript. This research project was supported by ENVISION, which is co-funded by the European Commission under FP7 Grant Agreement N. 241851, and by a Marie Curie Initial Training Network Fellowship of the European Communitys Seventh Framework Programme under contract number PITNGA2008-215840-PARTNER.

\section{A Lorentz and gauge transformations of polarized photons}

The state of a polarized photon, modelled as a point particle, is identified by its spacetime position $x^{\mu}$, its momentum vector $k^{\mu}=(k, \mathbf{k})$, and its polarization state $\varepsilon^{\mu}=\left(\varepsilon_{0}, \varepsilon\right)$ which fulfill the conditions

$$
k_{\mu} \varepsilon^{\mu}=0 \quad \text { and } \quad \varepsilon_{\mu} \varepsilon^{\mu}=1
$$

in all Lorentz frames. With a Lorentz transformation $\Lambda$ a polarized photon can be transformed to the rest frame of the electron with the electron momentum vector $p^{\mu}=\left(m_{\mathbf{e}} c, \mathbf{0}\right)$. It can be shown that an arbitrary Lorentz transformation results in a polar rotation about the momentum vector in the space of polarization. Details are given for instance in [39].

Being in the rest frame of the electron, $\varepsilon^{\mu}$ is defined apart from a gauge transformation [15]

$$
\bar{\varepsilon}^{\mu}=\varepsilon^{\mu}+\lambda k^{\mu}
$$

where $\lambda$ is a complex number, $\bar{\varepsilon}^{\mu}$ describes the same polarization state which still fulfills the conditions (A.1), as photons are massless particles with $k_{\mu} k^{\mu}=0$. When choosing a gauge in which the polarization vectors have only spatial components ('axial gauge' or 'transverse gauge' with $\bar{\varepsilon}_{0}=0$ ) we have additionally $p_{\mu} \bar{\varepsilon}^{\mu}=p_{\mu} \bar{\varepsilon}^{\prime \mu}=0$, where $\bar{\varepsilon}^{\prime \mu}$ is the polarization vector after a scattering process. Such a gauge transformation to $\bar{\varepsilon}_{0}=\bar{\varepsilon}_{0}^{\prime}=0$ is given by

$$
\lambda=-\frac{\varepsilon_{0}}{k} .
$$

\section{References}

[1] P. Eisenberger and P.M. Platzman, Compton scattering of $x$-rays from bound electrons, Phys. Rev. A 2 (1970) 415.

[2] M. Puska and R. Nieminen, Theory of positrons in solids and on solid surfaces, Rev. Mod. Phys. 66 (1994) 841 [INSPIRE].

[3] O. Klein and T. Nishina, Über die Streuung von Strahlung durch freie Elektronen nach der neuen relativistischen Quantendynamik von Dirac (in German), Z. Phys. 52 (1929) 853.

[4] H. Seo et al., Experimental performance of double-scattering Compton camera with anthropomorphic phantom, 2011 JINST 6 C01024.

[5] M. Rudin and R. Weissleder, Molecular imaging in drug discovery and development, Nat. Rev. Drug Discov. 2 (2003) 123.

[6] S.S. Gambhir, Molecular imaging of cancer with positron emission tomography, Nat. Rev. Cancer 2 (2002) 683 
[7] W. Enghardt et al., Charged hadron tumour therapy monitoring by means of PET, Nucl. Instrum. Meth. A 525 (2004) 284.

[8] K. Parodi et al., Patient study of in vivo verification of beam delivery and range, using positron emission tomography and computed tomography imaging after proton therapy, Int. J. Radiat. Oncol. 68 (2007) 920.

[9] P.J. Schultz and K. Lynn, Interaction of positron beams with surfaces, thin films and interfaces, Rev. Mod. Phys. 60 (1988) 701 [inSPIRE].

[10] P. Asoka-Kumar, M. Alatalo, V.J. Ghosh, A.C. Kruseman, B. Nielsen and K.G. Lynn, Increased elemental specificity of positron annihilation spectra, Phys. Rev. Lett. 77 (1996) 2097.

[11] V. Makarashvili and D.P. Wells, Deep defect density imaging, Nucl. Instrum. Meth. B 268 (2010) 2835.

[12] G. Battistoni et al., The FLUKA code: Description and benchmarking, in Proceedings of the Hadronic Shower Simulation Workshop 2006, Batavia U.S.A. (2006), AIP Conf. Proc. 896 (2007) 31.

[13] A. Ferrari, P.R. Sala, A. Fassò and J. Ranft, FLUKA: a multi-particle transport code, Technical Report CERN-2005-10 (2005).

[14] FLUKA collaboration, FLUKA Manual: http://www.fluka.org/ (2011).

[15] H. Kleinert, Particles and Quantum Fields, World Scientific, Singapore (1996).

[16] W. Heitler, The Quantum Theory of Radiation, Clarendon Press, Oxford U.K. (1936).

[17] I. Waller and D.R. Hartree, On the intensity of total scattering of $x$-rays, Proc. Roy. Soc. London A 124 (1929) 119.

[18] D.E. Cullen, J.H. Hubbell and L. Kissel, The Evaluated Photon Data Library, 97 version, UCRL-ID-50400, Lawrence Livermore National Laboratory 6 (1997).

[19] R. Ribberfors, Relationship of the relativistic Compton cross section to the momentum distribution of bound electron states, Phys. Rev. B 12 (1975) 2067.

[20] R. Ribberfors and K.-F. Berggren, Incoherent-x-ray-scattering functions and cross sections by means of a pocket calculator, Phys. Rev. A 26 (1982) 3325.

[21] D. Brusa, G. Stutz, J.A. Riveros, J.M. Fernández-Varea and F. Salvat, Fast sampling algorithm for the simulation of photon Compton scattering, Nucl. Instrum. Meth. A 379 (1996) 167.

[22] F. Biggs, L.B. Mendelsohn and J.B. Mann, Hartree-Fock Compton profiles for the elements, Atom. Data Nucl. Data 16 (1975) 201.

[23] J.H. Hubbell, Summary of existing information on the incoherent scattering of photons, particularly on the validity of the use of the incoherent scattering function, Radiat. Phys. Chem. 50 (1997) 113.

[24] M. Cooper, P. Pattison and J.R. Schneider, Compton profile measurements with $412 \mathrm{keV} \gamma$-radiation, Philosoph. Mag. 34 (1976) 243.

[25] P.W. Chin and N.M. Spyrou, Monte Carlo investigation of positron annihilation in medical positron emission tomography, Nucl. Instrum. Meth. A 580 (2007) 481.

[26] Y.-D. Jung, One-photon annihilation of thermal positrons with bound atomic electrons, Astrophys. J. 424 (1994) 988.

[27] K. Iwata, Positron Annihilation on Atoms and Molecules, Ph.D. Thesis, University of California, San Diego U.S.A. (1997). 
[28] A. Rich, Recent experimental advances in positronium research, Rev. Mod. Phys. 53 (1981) 127 [INSPIRE].

[29] L.S. Fornari, L.M. Diana and P.G. Coleman, Positronium formation in collisions of positrons with He, Ar, and $H_{2}$, Phys. Rev. Lett. 51 (1983) 2276.

[30] M. Alatalo et al., Theoretical and experimental study of positron annihilation with core electrons in solids, Phys. Rev. B 54 (1996) 2397.

[31] B. Barbiellini, M. Hakala, M.J. Puska, R.M. Nieminen and A.A. Manuel, Correlation effects for electron-positron momentum density in solids, Phys. Rev. B 56 (1997) 7136.

[32] S. Berko and J.C. Erskine, Angular distribution of annihilation radiation from plastically deformed aluminum, Phys. Rev. Lett. 19 (1967) 307.

[33] E. do Nascimento, O. Helene, V.R. Vanin and M.T.F. da Cruz, Statistical analysis of the Doppler broadening coincidence spectrum of the electron-positron annihilation radiation in aluminum, Nucl. Instrum. Meth. B 247 (2006) 38.

[34] R.P. Gupta and R.W. Siegel, On the determination of core and valence electron enhancement factors in positron annihilation studies, J. Phys. F 9 (1979) 2353.

[35] K.G. Lynn et al., Positron-annihilation momentum profiles in aluminum: Core contribution and the independent-particle model, Phys. Rev. Lett. 38 (1977) 241.

[36] K. Iwata, R.G. Greaves and C.M. Surko, $\gamma$-ray spectra from positron annihilation on atoms and molecules, Phys. Rev. A 55 (1997) 3586.

[37] D.G. Green, S. Saha, F. Wang, G.F. Gribakin and C.M. Surko, Effect of positron-atom interactions on the annihilation gamma spectra of molecules, New J. Phys. 14 (2012) 035021.

[38] K. Iwata, G.F. Gribakin, R.G. Greaves and C.M. Surko, Positron annihilation with inner-shell electrons in noble gas atoms, Phys. Rev. Lett. 79 (1997) 39.

[39] P. Caban and J. Rembielinski, Photon polarization and Wigner's little group, Phys. Rev. A 68 (2003) 042107. 